\title{
Nano and beyond: Fine tuning molecular interactions towards global sustenance
}

\author{
Mahesh Narayan \\ Department of Chemistry, The University of Texas at El Paso, El Paso, TX, U.S.A. \\ Email:mnarayan@utep.edu
}

Nanoparticle based applications and solutions have found widespread use in medicine, agriculture, water purification, environmental remediation, etc. Heretofore, the primary focus has remained that of enhancing potency of the agent through nano-sizing of the active ingredient to increase its "effectivity". Yet, collateral toxicity caused by nanoparticles is a burgeoning problem that is exacerbated by their inherently high "activity" resulting from high surface-tovolume ratios compared to other macrostructures.

Our laboratory is involved in fine-tuning ligandreceptor interactions using halogen bonding as an atomic scalpel [1]. We demonstrate that via haloinsertion and halo-shuffling it is possible to achieve enhanced binding efficiencies of ligands, improve specificity, and therefore eliminate or reduce their toxicity in the nanoengineered embodiment.

For example, we have examined the propensity of a host of halogen-bond-containing pharmaceuticals to bind to protein side-chain and backbone Lewis bases [1]. The data set was restricted to crystal structures deposited to date in the PDB. Data were analyzed as a function of halogen-bonding to main chain Lewis bases, viz. oxygen of backbone carbonyl and backbone amide nitrogen. This analysis also examined halogen bonding to side-chain Lewis bases $(\mathrm{O}, \mathrm{N}$, and $\mathrm{S}$ ) and to the electron-rich aromatic amino acids. All interactions were restricted to van der Waals radii with respective atoms. The results indicate that while fluorine and chlorine have strong tendencies favoring interactions with the backbone Lewis bases at glycine, the trend is not restricted to the achiral amino acid backbone for larger halogens. Halogen: side-chain interactions are not restricted to amino acids containing $\mathrm{O}, \mathrm{N}$, and $\mathrm{S}$ as Lewis bases. Electron-rich aromatic amino acids host a high frequency of halogen bonds as does Leu. A closer examination of the latter hydrophobic side chain reveals that the "propensity of interactions" of halogen ligands at this oily residue is an outcome of strong classical halogen bonds with Lewis bases in the vicinity. Finally, an examination of $\Theta 1(\mathrm{C}-\mathrm{X} \cdots \mathrm{O}$ and $\mathrm{C}$ $\mathrm{X} \cdots \mathrm{N})$ and $\Theta 2(\mathrm{X} \cdots \mathrm{O}-\mathrm{Z}$ and $\mathrm{X} \cdots \mathrm{N}-\mathrm{Z})$ angles

reveals that very few ligands adopt classical halogen bonding angles, suggesting that steric and other factors may influence these angles (Figure 1) [1].

Included examples are mechanism to fine-tune the binding of anti-depressants to their SSRI receptors. This is of environmental importance because the U.S. Geological Survey (USGS) has reported the presence of specific antidepressants and their degradates in wastewater discharged into streams by municipal wastewater treatment plants [2]. These chemotoxicants are taken up into the bodies of fish living downstream of the plants. The antidepressants were found in fish collected over 8 kilometers (approximately 5 miles) downstream of the location of the wastewater discharge. Several commonly used antidepressants were detected in water, streambed sediment, and the brain tissue of white suckers, a native fish species [2]. Fish collected upstream from the wastewater discharge did not have antidepressants present in their brain tissues.

It is proposed that such an emerging technology, i.e., the use of molecular fine-tuning via halogen bonding, will advance the nanotechnology frontier through molecular- and atomic-level intervention.

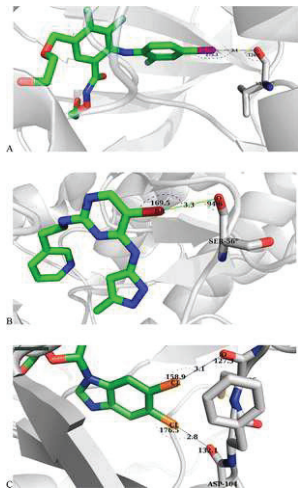

References

1. Sirimulla, S., Bailey, J. B., Vegesna, R., Narayan, M. Journal of Chemical Information and Modeling 53 (2013) 2781-91.

2. Schultz, M. M et al Environ. Sci. and Tech., 44 (2010) 1918-1925
Figure 1: Examples of halogen bonds in proteinligand complexes. (a) Ligand forming $\mathrm{I} \cdots \mathrm{O}$ interaction with carbonyl oxygen of Val 127 (pdb ID: $3 \mathrm{dv} 3$ ). (b) Ligand forming $\mathrm{Br} \cdots \mathrm{O}$ interaction with carbonyl oxygen of Ser 565. (c) Ligand forming $\quad \mathrm{Cl} \cdots \mathrm{O}$ interactions with Cys 106 and Asp 104. The image was prepared using PYMOL 